\title{
We Should Apply a “One Health" Approach to Understand and Treat Alzheimer's Disease
}

\author{
Anthony R. White \\ Mental Health Program, Department of Cell and Molecular Biology, QIMR Berghofer Medical Research Institute, Herston, Qld, \\ Australia \\ Email: Tony.White@qimrberghofer.edu.au
}

How to cite this paper: White, A.R. (2021) We Should Apply a "One Health" Approach to Understand and Treat Alzheimer's Disease. Advances in Alzheimer's Disease, 10, 46-51.

https://doi.org/10.4236/aad.2021.103004

Received: July 30, 2021

Accepted: September 6, 2021

Published: September 9, 2021

Copyright (c) 2021 by author(s) and Scientific Research Publishing Inc. This work is licensed under the Creative Commons Attribution International License (CC BY 4.0).

http://creativecommons.org/licenses/by/4.0/

\section{Open Access}

\begin{abstract}
Despite many decades of researches and large numbers of clinical trials, there remain no effective treatments for Alzheimer's disease, a major degenerative ageing brain disorder. The potential treatments have focused on targeting the accumulation of amyloid beta-peptide in the brains of patients, but without success in slowing the disease. Many studies have now identified a large range of pathological changes (i.e. altered immune activity, mitochondrial impairment, abnormal microbiome), and links to the external environment (i.e. associations with infections, the influence of air pollution). While the concept of One Health (which considers links between the environment and human disease) has traditionally been applied to the understanding of the human infectious disease, it is argued here that the One Health approach should be adopted for Alzheimer's disease. This would provide a far more holistic understanding of the disease, and its relationship to a growing number of exogenous factors, as well as could potentially lead to new treatment options targeted at the confluence of external influences, and internal molecular pathways.
\end{abstract}

\section{Keywords}

One Health, Alzheimer's Disease, Multifactorial Disease

\section{Introduction}

The term "One Health" was developed in 2004 in relation to human infectious diseases such as SARS and H5N1 Influenza-associated with anthropogenic impact on wildlife and ecosystems. The concept started as One Medicine, then One World One Health and more recently, simply One Health. One Health "recognizes that the health of humans, animals and ecosystems are interconnected. It involves applying a coordinated, collaborative, multidisciplinary and cross-sectoral 
approach to address potential or existing risks that originate at the animal-human-ecosystems interface" [1].

While this concept has traditionally been applied to the integral and complex relationship between the environment (animal, plant, and disease agents and vectors), and human health (primarily infectious disease) [2] [3], there is a strong argument for the application of this concept to diseases that have traditionally (but perhaps incorrectly) been attributed more to an intrinsic genetic basis such as cancer and neurological disease. The rationale for extending a One Health approach to complex, and multifactorial diseases, such as Alzheimer's disease, a major ageing disorder, is the growing realization that such diseases do in fact have many pathological changes and etiological links to environmental factors.

\section{Alzheimer's Disease}

Alzheimer's Disease (AD) is the most common neurodegenerative disorder and affects over 50 million people worldwide. AD patients suffer progressive cognitive decline, with death normally occurring between 3-9 years after diagnosis. Due to an ageing population, numbers of $\mathrm{AD}$ cases are expected to reach 150 million by 2050. Despite over 100 years of investigation and over 160,000 scientific papers published, there has been little advance in treatment of the disease. Over 200 clinical trials have failed, and there are no disease-modifying drugs approved for treating patients (only symptomatic treatment). The last approved drug for AD was in 2003, nearly 20 years ago. Aduhelm may change this, although it is yet to clear a Phase 4 trial [4]. Clearly, targeting a single feature of $\mathrm{AD}$, such as amyloid aggregation, while potentially yet offering some small benefit in a subset of patients, will not lead to a broad preventative or cure for such a complex disease.

\section{Rethinking Our Approach to Alzheimer's Disease}

It is this complexity that not only makes treatment approaches difficult, it also underlines the basis for re-thinking our approach, and adopting a One Health interpretation. This complexity can be seen when we look at the pathological hallmarks of AD, which extend well beyond accumulation of amyloid and tau proteins (the hallmark neuropathology). Growing evidence now paints a complex multifactorial picture of the disease that includes strong changes to the immune system both centrally and peripherally, a relationship between gut, and possibly other microflora and the disease, traumatic brain injury, mitochondrial dysfunction, olfactory changes, oxidative stress, altered trace element and biometal homeostasis, links to external factors such as air pollution (including industrial and domestic), and a strong link between infectious disease agents (viruses, bacteria, and parasites) and $\mathrm{AD}$ [5] [6] [7]. All these must be integrated into the growing understanding of polygenic backgrounds, diet, sleep, exercise, mental health, vascular disease, diabetes, and of course ageing, and other factors as reviewed in [8] (Figure 1). Whilst examining the impact of each of these fac- 
tors independently is already an enormous challenge (i.e. over 8000 papers already exist on inflammation and $\mathrm{AD}$ ) many (perhaps all) of these factors show complex integration. For example, the immune system is modulated by microbiomes, state of mental health, biometal levels, diet, sleep, environmental pollution, infections, etc. [9] [10]. And conversely, many of these factors can also potentially be affected by the immune system. Even if we restricted the major factors affecting $\mathrm{AD}$ onset and progression to $10-20$ (this is likely to be a very low estimate), if each of those also affects and is affected by each of the other factors, the combination of these interplays becomes perhaps as complex as trying to understand the functioning of an entire ecological system, certainly supporting the concept of needing to approach $\mathrm{AD}$ with a One Health mindset.

The One Health approach for $\mathrm{AD}$ is further supported by the growing recognition that our health, including brain disorders, is closely linked to the environmental world. This is evident not just through the strong evidence for infectious disease agents in $\mathrm{AD}$ [11], but the strong relationship between $\mathrm{AD}$ and other environmental factors including air pollution, biometals (which come

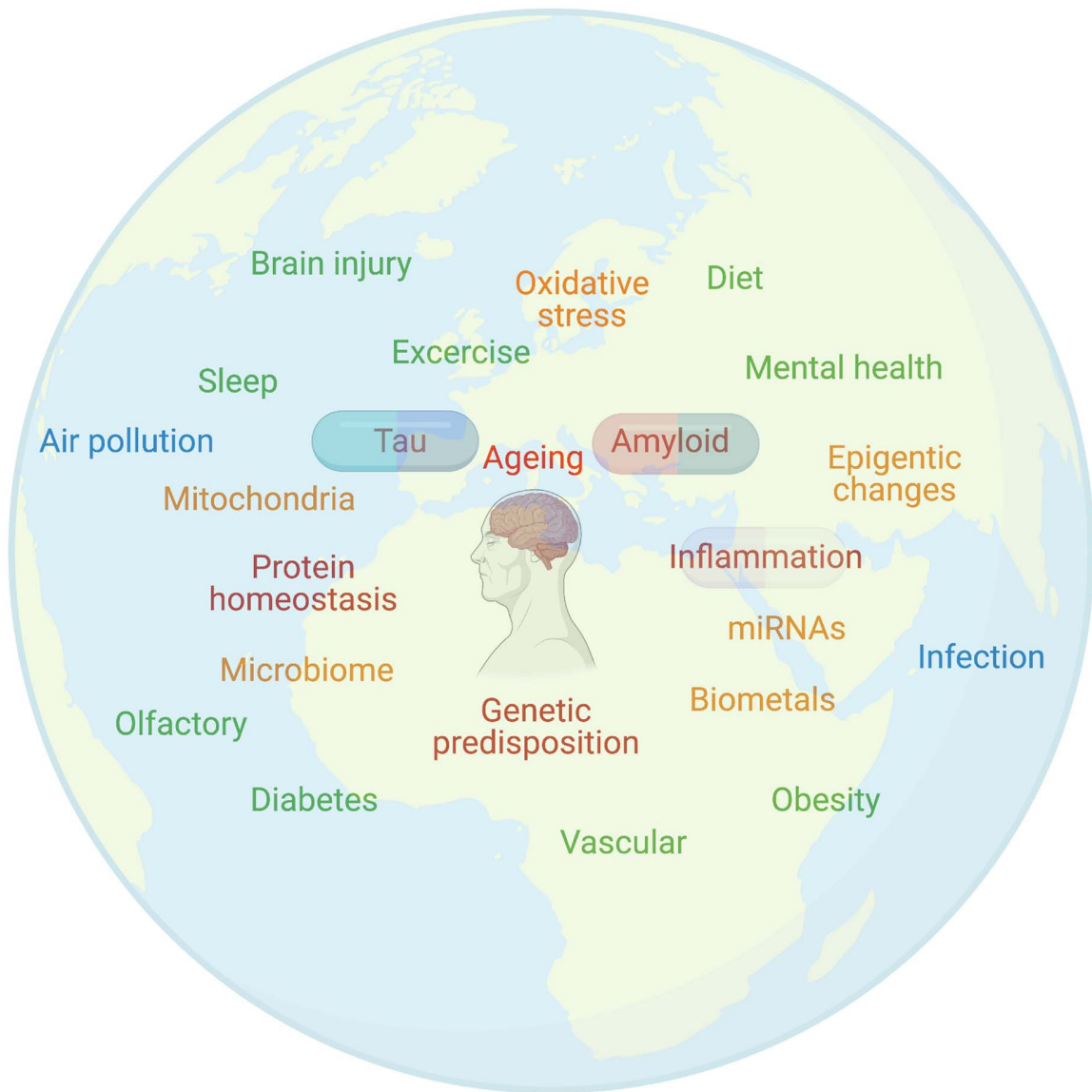

Figure 1. Alzheimer's disease consists of several major pathological elements (red), additional processes that link the endogenous changes to the external environment (orange), a number of contributing factors that are again linked to external influences (green), and finally truly exogenous impacts (blue). The only factors in the disease currently being targeted at a significant level are amyloid, tau, and now inflammation. 
from the bioinorganic world) [12] [13] [14], impact of heat stress (driven more recently by climate change), and even access to green space and its influence on mental and physical health [15]. The influence of these factors may be direct, or can modulate our susceptibility to, and progression of, $\mathrm{AD}$, via polygenic relationships, or epigenetics. If a human disease is impacted by a broad range of environmental factors, such as occurs with $\mathrm{AD}$, then surely, we should try to understand the disease using a One Health approach.

\section{A One Health Approach to Alzheimer's Disease Will Change How We Study and Treat the Disorder}

So, assuming that we undertake this approach, what would that mean for future studies and treatments for $\mathrm{AD}$ ? Primarily, we should not think of $\mathrm{AD}$ as a disease caused by protein accumulation alone. This has not worked after decades of intense research and billions of research dollars. We must also accept that there will be no single effective treatment or cure for $\mathrm{AD}$, or any complex neurological disease. Simply targeting one aspect of the disease is akin to plugging a single hole in a sieve and expecting it to stop the water flow. We must have a greater focus on cross-disciplinary research, and encourage researchers to examine how multiple factors interplay in the cause and progression of disease. Clearly no one group can simply tie together all the pieces we know contribute to the disease, just as no one group can understand all aspects of an ecological system or how it contributes to human zoonoses such as SARS-CoV-2. However, large multi-disciplinary teams can make enormous progress finding links between key etiological and pathological factors of disease, and in future, other teams can then tie these "nodes" together to form networks that underlie disease. This approach can lead to identification of disease "hubs" that could be targeted to have broad influence over a number of different pathological changes. Such research requires the synergy of teams that are truly multi-disciplinary with expertise from molecular analysis through to the clinic, population studies, genetics, bioinformatics, and drug targeting. This concept of network targeting generally does not appeal to pharmaceutical companies, which prefer to back one drug-one target options. However, these have universally failed in $\mathrm{AD}$ for reasons outlined above. There is little to be lost by trying to control a disease at a pathological "ecosystem" level, like restoring an apex predator in a field ecosystem that leads to ripples of changes across that system. Incentive from governments should be focused on bringing together multidisciplinary teams and the resources of pharma to explore highly innovative targeting of major hubs and/or multiple targets in $\mathrm{AD}$.

We also need to encourage researchers, and teachers to adopt a broader understanding of the disease. Attend any $\mathrm{AD}$ conference, or read an $\mathrm{AD}$ review paper and invariably the description of the pathological basis will rarely extend beyond clinical symptoms, amyloid and tau accumulation, together with a more detailed description of the researcher's topic of interest. For example, there are over 6,000 papers on metals and $\mathrm{AD}$, yet very few $\mathrm{AD}$ presentations or generalist 
reviews mention metals at all. Such narrow approaches to describing AD help to perpetuate the concept that $\mathrm{AD}$ is a relatively simple disease driven by amyloid and tau accumulation with a few secondary elements. As described above, this understanding has not led to an effective drug in clinical trials to date. Such a concept would be akin to teaching ecology students that a forest ecosystem consists only of trees. Finally, we need to appreciate and support the acceptance that $\mathrm{AD}$ is not a disease caused by a single intrinsic pathology, i.e. abnormal protein handling, but a complex brain disorder that sits in the centre of a web linking many aspects of the external world, and peripheral organs and tissues.

\section{Conclusion}

There are signs that some funding organizations are responding to the One Health challenge of $\mathrm{AD}$, asking for high-risk, innovative applications that support multi-disciplinary teams (such as the Joint Programme-Neurodegenerative Disease Research, JPND). Moreover, research from multi-disciplinary teams tends to have a higher impact than teams with a narrow focus. We hope that these factors will translate into an increase in research papers and review articles that capture the complexity of $\mathrm{AD}$, its integral relationship with the natural world, and a growing recognition that successful management of the disease will require targeting multiple pathological pathways and/or key hubs that control many aspects of the disease. Just as with the growing understanding of One Health in ecology and human infection, we cannot continue to look at $\mathrm{AD}$ as a disease that is separate from the continuum of external and internal factors that drive its aetiology over years to decades in people that suffer from the disease.

\section{Acknowledgements}

Figure prepared using Biorender. ARW is supported by a National Health and Medical Research Council of Australia Senior Research Fellowship.

\section{Conflicts of Interest}

The author declares no conflicts of interest regarding the publication of this paper.

\section{References}

[1] Mackenzie, J.S. and Jeggo, M. (2019) The One Health Approach-Why Is It So Important. Tropical Medicine and Infections Disease, 4, 88. https://doi.org/10.3390/tropicalmed4020088

[2] King, T.A. (2021) The One Medicine Concept: Its Emergence from History as a Systematic Approach to Re-Integrate Human and Veterinary Medicine. Emerging Topics in Life Sciences, ETLS20200353. https://doi.org/10.1042/ETLS20200353

[3] Solis, A. and Nunn, C.L. (2021) One Health Disparities and COVID-19. Evolution, Medicine, and Public Health, 9, 70-77. https://doi.org/10.1093/emph/eoab003

[4] Mukhopadhyay, S. and Banerjee, D. (2021) A Primer on the Evolution of Aducanumab: The First Antibody Approved for Treatment of Alzheimer's Disease. Journal of Alzheimer's Disease, 1-16. https://doi.org/10.3233/JAD-215065 
[5] Ju, Y. and Tam, K.Y. (2022) Pathological Mechanisms and Therapeutic Strategies for Alzheimer's Disease. Neural Regeneration Research, 17, 543-549. https://doi.org/10.4103/1673-5374.320970

[6] Das, N., Raymick, J. and Sarkar, S. (2021) Role of Metals in Alzheimer's Disease. Metabolic Brain Disease. https://doi.org/10.1007/s11011-021-00765-w

[7] Behl, T., Makkar, R., Sehgal, A., Brisc, C., et al. (2021) Current Trends in Neurodegeneration: Cross Talks between Oxidative Stress, Cell Death, and Inflammation. International Journal of Molecular Sciences, 22, 7432. https://doi.org/10.3390/ijms22147432

[8] Qiu, C., Kivipelto, M. and von Strauss, E. (2009) Epidemiology of Alzheimer's Disease: Occurrence, Determinants, and Strategies toward Intervention. Dialogues in Clinical Neurosciences, 11, 111-128. https://doi.org/10.31887/DCNS.2009.11.2/cqiu

[9] Calder, P.C., Bosco, N., Bourdet-Sicard, R., Visioli, F., et al. (2017) Health Relevance of the Modification of Low Grade Inflammation in Ageing (Inflammageing) and the Role of Nutrition. Ageing Research Reviews, 40, 95-119. https://doi.org/10.1016/j.arr.2017.09.001

[10] Uniyal, A., Tiwari, V., Rani, M., Tiwari, V., et al. (2021) Immune-Microbiome Interplay and Its Implications in Neurodegenerative Disorders. Metabolic Brain Disease. https://doi.org/10.1007/s11011-021-00807-3

[11] Sochocka, M., Zwolińska, K. and Leszek, J. (2017) The Infectious Etiology of Alzheimer's Disease. Current Neuropharmacology, 15, 996-1009. https://doi.org/10.2174/1570159X15666170313122937

[12] Fu, P. and Yung, K.K.L. (2020) Air Pollution and Alzheimer's Disease: A Systematic Review and Meta-Analysis. Journal of Alzheimer's Disease, 77, 701-714. https://doi.org/10.3233/JAD-200483

[13] Acevedo, K., Masaldan, S., Opazo, C.M., Bush, A.I., et al. (2019) Redox Active Metals in Neurodegenerative Diseases. Journal of Biological Inorganic Chemistry, 24, 1141-1157. https://doi.org/10.1007/s00775-019-01731-9

[14] Wu, J. and Jackson, L. (2021) Greenspace Inversely Associated with the Risk of Alzheimer's Disease in the Mid-Atlantic United States. Earth, 2, 140-150. https://doi.org/10.3390/earth2010009

[15] Astell-Burt, T., Navakatikyan, M.A. and Feng, X. (2020) Urban Green Space, Tree Canopy and 11-Year Risk of Dementia in a Cohort of 109,688 Australians. Environment International, 145, Article No. 106102.

https://doi.org/10.1016/j.envint.2020.106102 